\title{
Anatomia microcirúrgica das artérias infratentoriais: um estudo estereoscópico
}

\author{
Microsurgical anatomy of the infratentorial arteries: stereoscopic printing study \\ Gustavo Rassier Isolan', Adamastor Humberto Pereira 1,2, Paulo Henrique Pires de Aguiar', \\ Ápio Cláudio Martins Antunes ${ }^{1,3}$, João Pedro Mousquer³, Marcel Rozin Pierobon ${ }^{1}$
}

\section{Resumo}

Contexto: O espaço infratentorial do crânio é uma região que possui complexa rede de artérias que vascularizam o tecido cerebelar e o tronco encefálico. Sua complexa anatomia tridimensional deve ser profundamente entendida pelo neurocirurgião vascular e pelo radiologista intervencionista. Objetivo: Descrever as principais artérias do espaço infratentorial e seu trajeto, bem como sua relação com a topografia das estruturas neurais. Métodos: Foram estudados 30 cérebros humanos, fixados em formalina e 6 cabeças de cadáveres dissecadas, utilizando-se microscópico cirúrgico com 3X a 40X de aumento. Os cérebros e todas as cabeças foram injetados com siloxanos polimerizados ou polisiloxanos (silicone) colorido. As dissecções anatômicas foram documentadas utilizando-se técnica para obtenção de imagens tridimensionais (3D), objetivando a produção de impressões estereoscópicas.

Resultados: São descritos o trajeto, segmentos e ramos das artérias basilar, cerebelar superior, cerebelar anteroinferior, cerebelar posteroinferior e vertebral. Conclusão: A anatomia das artérias infratentoriais é complexa e as imagens estereoscópicas apresentadas são um importante instrumento de documentação, pois permitem uma noção de profundidade da anatomia estudada.

Palavras-chave: artérias cerebrais; microcirurgia; visão estereoscópica.

\begin{abstract}
Background: The infratentorial space is a region with a complex network of arteries supplying the cerebellum and brainstem. Its complex threedimensional anatomy must be thoroughly understood by the vascular neurosurgeon and the interventional radiologist.

Objective: To describe the main arteries of the infratentorial space and its trajectory and its relationship to the topography of the neural structures. Methods: We studied 30 formalin-fixed human brains and we also dissected 6 cadaver heads, using a surgical microscope with $3 X$ to $40 X$ magnification. The brains and all heads were injected with colored silicone. The anatomical dissections were documented with a three-dimensional method, aiming to produce stereoscopic prints.

Results: The neurovascular relationships, segments and branches of the basilar artery, superior cerebellar, anteroinferior cerebellar, cerebellar and posteroinferior vertebral are described.

Conclusion: The microsurgical anatomy of the infratentorial arteries is complex and the stereoscopic images presented are an important tool for documentation.

Keywords: cerebral arteries; microsurgery; stereoscopic vision.

\section{Introdução}

O compartimento infratentorial é um espaço intracraniano localizado abaixo da tenda do cerebelo. Na prática, confunde-se a denominação infratentorial com fossa posterior do crânio ${ }^{1}$. Esta última é o compartimento formado quase que inteiramente pelo osso occipital e separado

anteriormente da fossa média pela parte petrosa do osso temporal e o dorso da sela do esfenóide ${ }^{1}$. Ali são encontradas todas as estruturas encefálicas que não correspondem ao prosencéfalo, ou seja, mesencéfalo, metencéfalo (cerebelo e ponte) e mielencéfalo (bulbo). Destas estruturas, emerge uma série de nervos responsáveis pelas principais integrações sensitivas entre o indivíduo e meio ambiente ${ }^{1}$.
\end{abstract}

\footnotetext{
Trabalho realizado no Laboratório de Microcirurgia da Universidade de Arkansas - Little Rock (AR), EUA.

${ }^{1}$ Pós-graduação em Ciências Cirúrgicas da Universidade Federal do Rio Grande do Sul - Porto Alegre (RS), Brasil.

${ }^{2}$ Serviço de Cirurgia Vascular do Hospital de Clínicas de Porto Alegre - Porto Alegre (RS), Brasil.

${ }^{3}$ Serviço de Neurocirurgia do Hospital de Clínicas de Porto Alegre - Porto Alegre (RS), Brasil.

Conflito de interesse: nada a declarar.

Submetido em: 17.07.10. Aceito em: 26.09.11.

J Vasc Bras. 2012;11(2):114-122.
} 
Como representa uma considerável parte da base do crânio, a fossa posterior possui uma série de pequenos forames ósseos por onde transitam nervos cranianos (no caso da fossa posterior, são os forames correspondentes aos nervos cranianos VII, VIII, IX, X, XI e XII) e vasos sanguíneos ${ }^{2}$. Do ponto de vista anatômico-cirúrgico, a principal característica dessa região da base do crânio em relação às outras é a comunicação estabelecida aqui entre o encéfalo e a medula ${ }^{2}$.

A existência dos principais pontos de comunicação entre as estruturas intracranianas, medula e o resto do organismo é fornecida principalmente pela existência do forame magno, onde as vias de integração sensitivo-motoras deixam a cavidade craniana, e pelo forame jugular, o qual é o responsável pela drenagem de sangue venoso do sistema nervoso central ${ }^{3}$. As microestruturas, aqui presentes, são representadas pelos nervos cranianos (todos, exceto pelos dois primeiros nervos), que se originam do tronco encefálico e se dirigem aos forames de onde sairão do crânio ${ }^{3}$. Esse grande número de estruturas delicadas e vitais, circulando num espaço tão reduzido, acaba por tornar a abordagem cirúrgica dessa região extremamente cautelosa. Este artigo visa principalmente esclarecer, de maneira geral, o trajeto e a relação das artérias deste compartimento e sua relação com outras estruturas adjacentes.

As artérias que suprem esta região são oriundas do sistema arterial vértebro-basilar, um sistema proveniente de ramos das artérias vertebrais e que tem mais variações anatômicas quando comparado ao sistema carotídeo ${ }^{4}$. Seus principais vasos são as artérias cerebelares e as artérias cerebrais posteriores. Estes vasos organizam-se com as estruturas da fossa posterior formando três complexos neurovasculares, formados pelas artérias cerebelares superiores (ACS), artérias cerebelares inferiores anteriores (ACIA) e artérias cerebelares inferiores posteriores (ACIP) ${ }^{4}$.

Neste artigo, optou-se por descrever esta anatomia com bases nos estudos de Rothon ${ }^{5,6}$ e Yasargil ${ }^{7}$, em dissecções realizadas durante o período de dois anos e meio, em dois diferentes laboratórios de microcirurgia.

\section{Material e Métodos}

Este artigo é o resultado das dissecções realizadas por um dos autores (GRI) durante o período de dois anos e meio de pesquisa, em dois diferentes laboratórios de microcirurgia. As primeiras dissecções foram realizadas no Laboratório de Microcirurgia do Hospital Beneficência Portuguesa de São Paulo - Instituto de Ciências Neurológicas, durante período de um ano. A segunda parte desse estudo foi desenvolvida no Microsurgical Laboratory Diane and Gazi
Yaşargil Education Center - University of Arkansas for Medical Sciences, durante um período de 16 meses.

Trinta cérebros humanos fixados em formalina e seis cabeças de cadáveres foram dissecados utilizando-se de um microscópico cirúrgico com $3 \mathrm{X}$ a $40 \mathrm{X}$ de aumento. Nos cérebros e em todas as cabeças, foram injetados siloxanos polimerizados ou polisiloxanos (silicone) coloridos. Os cadáveres foram posicionados em um fixador de crânio, do tipo Mayfield, sendo a cabeça estendida e rotada, simulando o posicionamento cirúrgico, sendo, a seguir, realizadas as craniotomias petrosa e suboccipital.

As dissecções anatômicas foram documentadas utilizando-se técnica para obtenção de imagens tridimensionais (3D), objetivando a produção de impressões estereoscópicas. Nesta técnica, o mesmo objeto é fotografado a partir de duas posições diferentes, porém em um mesmo plano horizontal. A primeira corresponde à visão do olho esquerdo e a segunda à do olho direito. As imagens foram superpostas com software previamente definido, coordenadas e impressas. A utilização de óculos 3D com lentes coloridas é necessária para visualização das imagens impressas. Com a finalidade de documentar as dissecções foi utilizada uma câmera digital Nikkon D70 de 8,0 megapixel com lente macro. O aparelho foi adaptado em uma barra deslizante montada em um tripé. A lente e a velocidade de disparo foram ajustadas em f32 e 1/60 segundos, respectivamente. A técnica de estereoscopia utilizada pelos autores encontra-se detalhada em estudo prévio de Ribas, Bento e Rodrigues ${ }^{8}$.

As fotos impressas no método estereoscópico devem ser visualizadas com óculos estereoscópicos nas cores vermelha e verde.

\section{Resultados}

As Figuras 1 a 9 ilustram a anatomia microcirúrgica das artérias infratentoriais.

\section{Artéria vertebral}

A artéria vertebral cursa na região cervical, dentro dos forames transversos. Após deixar o processo transverso da primeira vértebra cervical, a artéria vertebral faz um giro em direção medial e adentra na fossa posterior através da membrana atlanto-occipital ${ }^{9,10}$. Em seu trajeto intracraniano inicial, esta artéria passa pela cisterna cerebelo-medular lateral, atinge o sulco bulbo-pontino, onde se une à sua correspondente do lado oposto para formar a artéria basilar ${ }^{9,10}$. Geralmente, esta união ocorre ao nível do terço inferior do clivo. Os ramos da artéria vertebral incluem vários pequenos ramos para as porções anterolateral, lateral e 
posterolateral da medula, a artéria espinhal anterior e, em mais de $70 \%$ dos casos, a artéria cerebelar posteroinferior ${ }^{9,10}$ (Figuras 1, 4 e 9).

\section{Artéria basilar}

A artéria basilar $(\mathrm{AB})$ tem sua origem no sulco bulbo-pontino onde as duas artérias vertebrais se unem. Ela cursa na cisterna pré-pontina em um sulco (sulco para a artéria basilar) na linha média da ponte. Seu segmento terminal alcança a cisterna interpeduncular, onde se divide nas duas artérias cerebrais posteriores ao nível do dorso da sela turca ${ }^{11-13}$. O curso desta artéria é tortuoso na maioria da população. Fenestração na artéria basilar pode ser encontrada em $1 \%$ dos casos. Os ramos que têm origem na $\mathrm{AB}$ são as artérias perfurantes circunferenciais e paramedianas que suprem a maior parte da ponte e do mesencéfalo. Os ramos maiores que têm origem na $\mathrm{AB}$ são a ACIA e a $\operatorname{ACS}^{11-13}$ (Figuras 1 e 4 ).

\section{Artéria cerebelar superior}

A ACS é a mais constante (tanto quanto à sua presença, como em relação à sua distribuição e curso) das artérias infratentoriais. Ela emerge da artéria basilar, próximo de seu topo, no ponto de bifurcação, perto do nervo oculomotor, seguindo em direção posterolateral contornando os limites entre ponte e pedúnculo cerebral ${ }^{14,15}$. Passa acima da origem do nervo trigêmeo e troclear e penetra na fissura cerebelo-mesencefálica, onde dá origem a uma série de pequenas artérias (artérias précerebelares). Seu tronco principal se divide em dois, os quais geralmente são de tamanhos iguais. Um deles percorre a superfície superolateral do cerebelo (tronco caudal) e, o outro, mais profundo, irrigará o vermis (tronco rostral).

De acordo com a sua posição em relação às estruturas em seu trajeto, essa artéria se divide em quatro segmentos: ponto-mesencefálico anterior e lateral, cerebelo-mesencefálico e cortical ${ }^{14,15}$ (Figuras 1, 5-7).

\section{Segmento ponto-mesencefálico anterior}

Este segmento começa na origem da ACS, no ápice da artéria basilar, normalmente, medial à borda livre da tenda do cerebelo; em seguida, passa pelos nervos cranianos III, IV e V, e segue até a margem anterolateral do tronco.

A junção ponto-mesencefálica é onde mais comumente ocorre a bifurcação da ACS. Pontos de bifurcação, diferentes deste, servem como referência para a localização de uma origem menos comum da $\mathrm{ACS}^{16,17}$. Geralmente, bifurcações anteriores ao mesencéfalo são associadas com ACS de origem supratentorial e bifurcações anteriores à ponte, com origens infratentorial.

O tronco principal da ACS pode emitir ramos perfurantes, que podem ser do tipo direto ou circunflexo. O ramo mais comum com origem do tronco principal é um ramo circunflexo longo. Esses ramos perfurantes, habitualmente, terminam no tegumento, na junção dos pedúnculos cerebelares superior e médio e na fossa interpeduncular ${ }^{16,17}$.

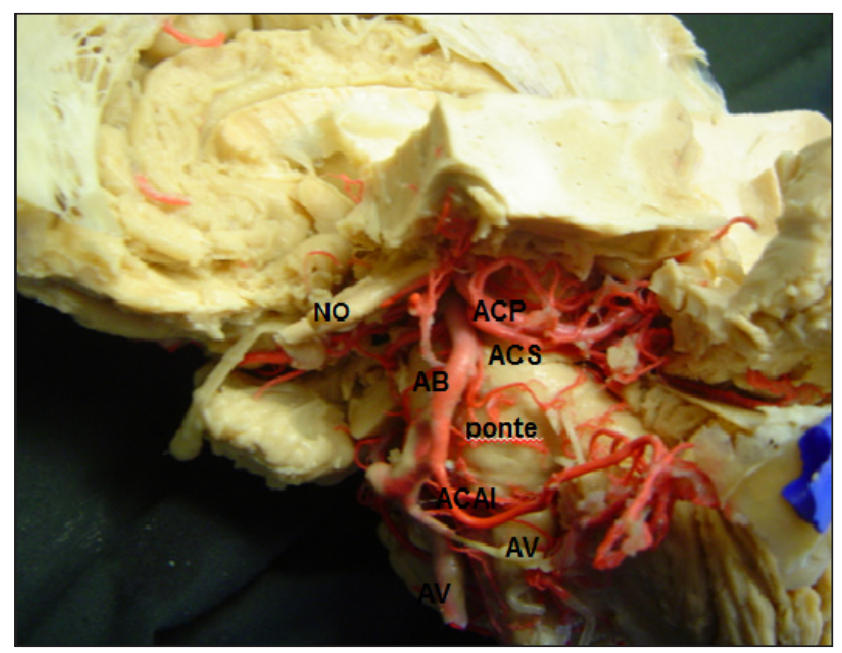

Figura 1. Visão lateral das artérias e fossa posterior. AV: artéria vertebral; AB: artéria basilar (observa-se fenestração); ACIA: artéria cerebelar inferior anterior; NO: nervo óptico; ACS: artéria cerebelar superior; ACP: artéria cerebral posterior.

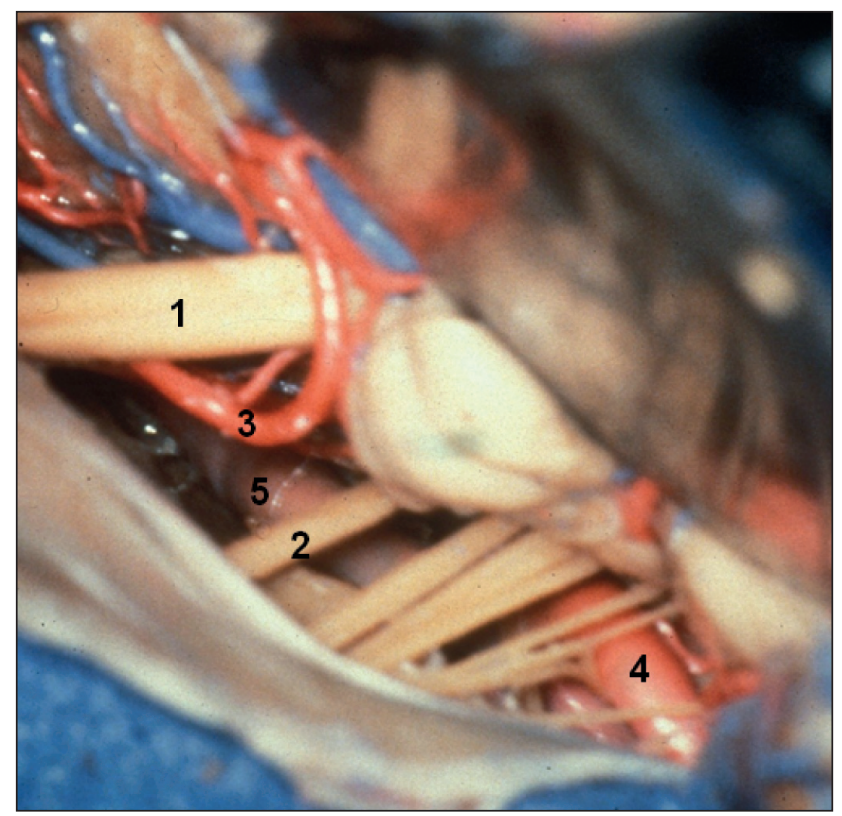

Figura 2. Visão das artérias infratentorias, através da abordagem suboccipital. (1) Complexo do VII e VIII nervos cranianos; (2) nervo glossofaríngeo; (3) artéria cerebelar anterior inferior; (4) artéria vertebral; (5) artéria basilar. 


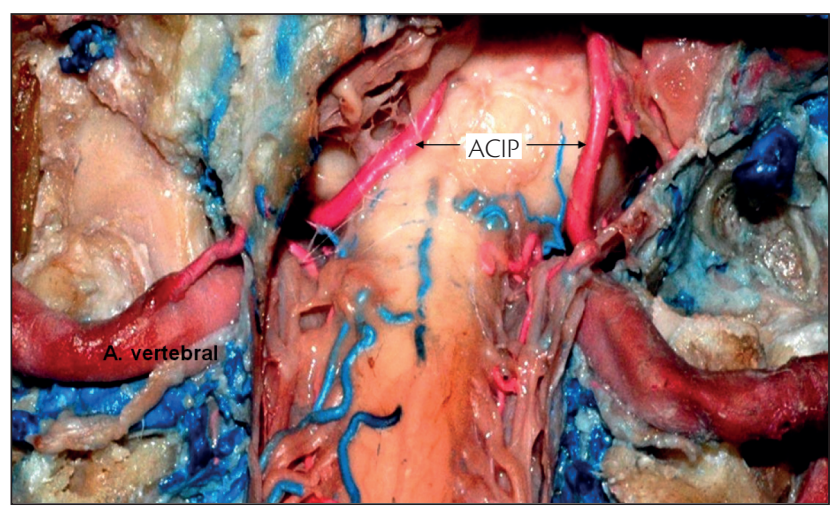

Figura 3. Visão posterior das artérias vertebral e da artéria cerebelar inferior posterior.

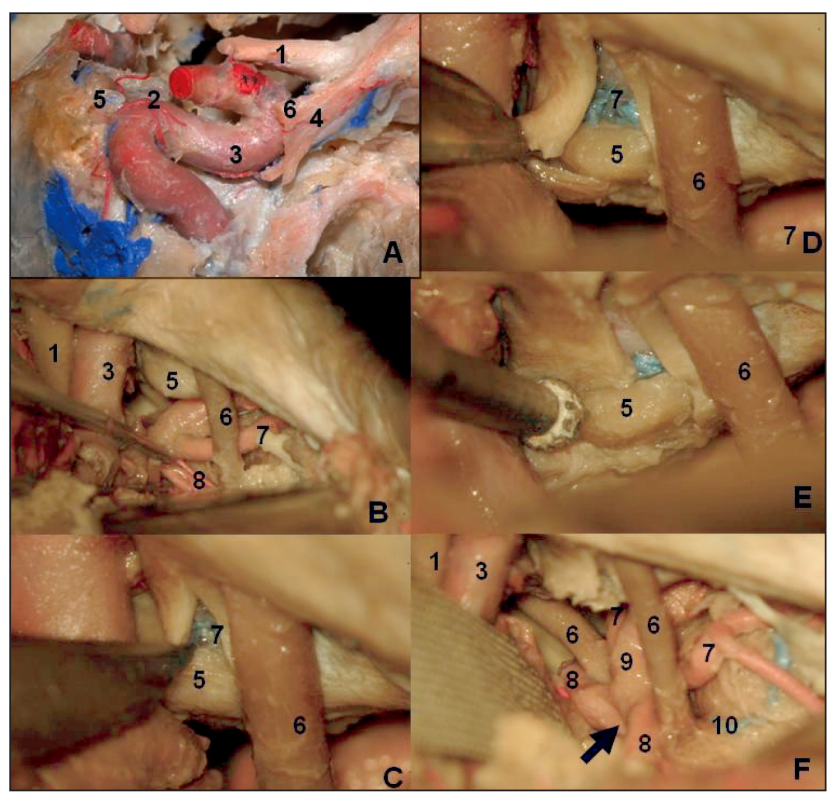

Figura 4. Visualização micro-anatômica dos passos da abordagem trans-silviana transcavernosa para o topo da artéria basilar. (A) Relação do tronco meningo-hipofisário originando-se da porção intracavernosa da artéria carótida interna (2) com o processo clinoide posterior (5); (B) nota-se a espátula de cérebro retraindo posteriormente o lobo temporal direito (acesso pré-temporal) para evidenciar (após dissecção da aracnoide das cisternas da base) a artéria basilar; (C) abertura do triângulo óculo-motor (teto do seio cavernoso) medialmente ao nervo óculo-motor; (D) exposição da clinoide posterior; (E) drilagem da clinoide posterior; (F) exposição do topo da artéria basilar (seta) bem como de seus ramos terminais: (1) nervo óptico; (2) tronco meningo-hipofisário; (3) artéria carótida interna; (4) ramo oftálmico do nervo trigêmio; (5) processo clinoide posterior; (6) nervo óculo-motor; (7) artéria cerebelar superior; (8) artéria cerebral posterior; (9) artéria basilar.

\section{Segmento ponto-mesencefálico lateral}

O segmento ponto-mesencefálico lateral é definido como aquele que começa após a emergência do nervo

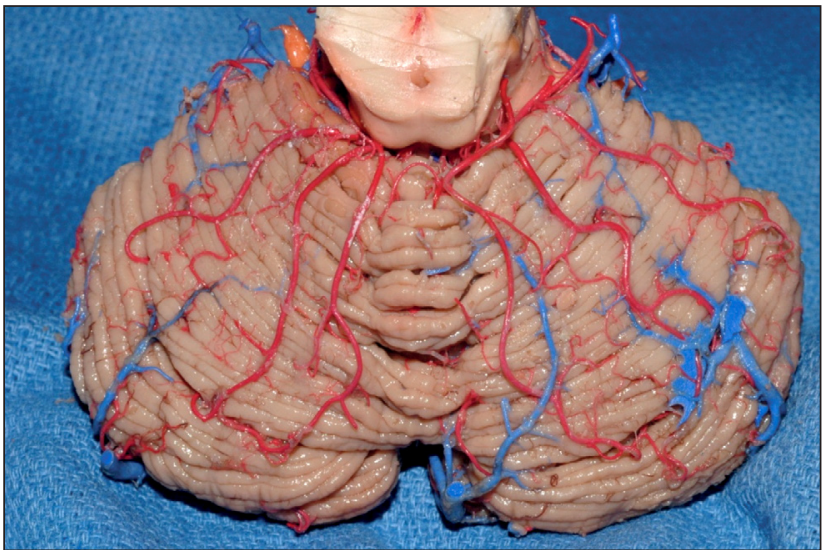

Figura 5. Ramos da artéria cerebelar superior. Nessa dissecção anatômica, observam-se os ramos da artéria cerebelar superior sobre a superfície superior do cerebelo.

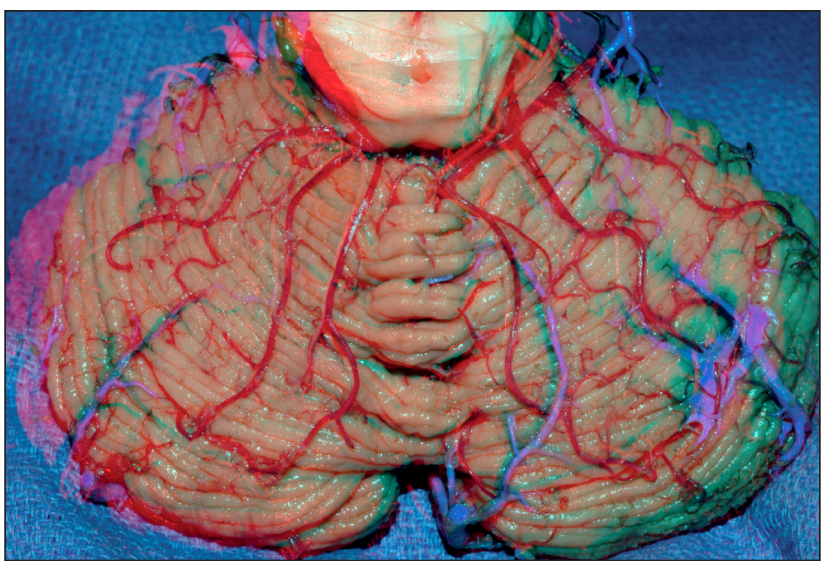

Figura 6. Visão estereoscópica dos ramos da artéria cerebelar superior.

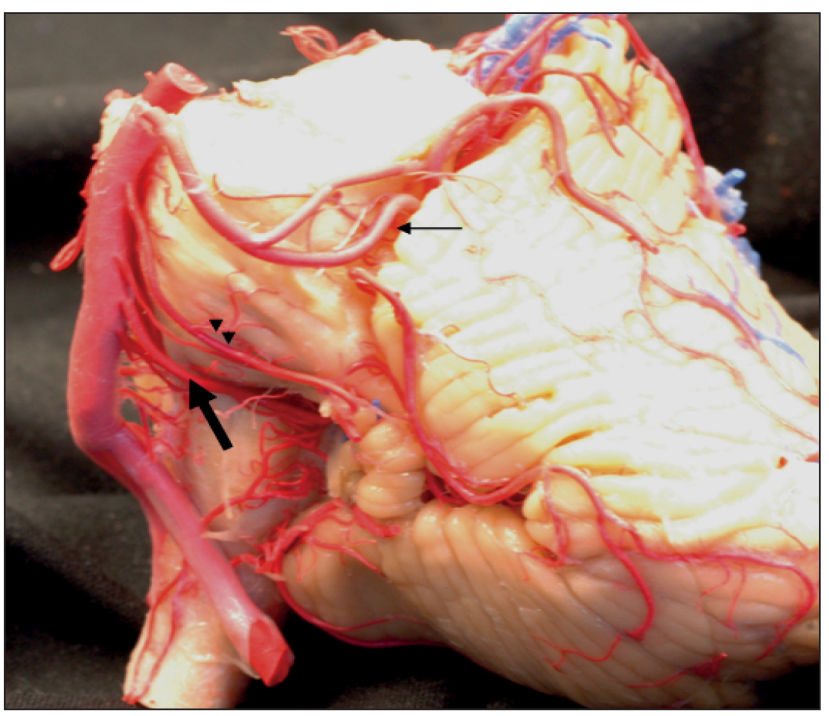

Figura 7. Peça anatômica mostrando os ramos da artéria basilar artéria cerebelar superior (seta), artéria cerebelar anterior inferior (pontas de seta) e ramos perfurantes e circunferenciais (seta espessa). 


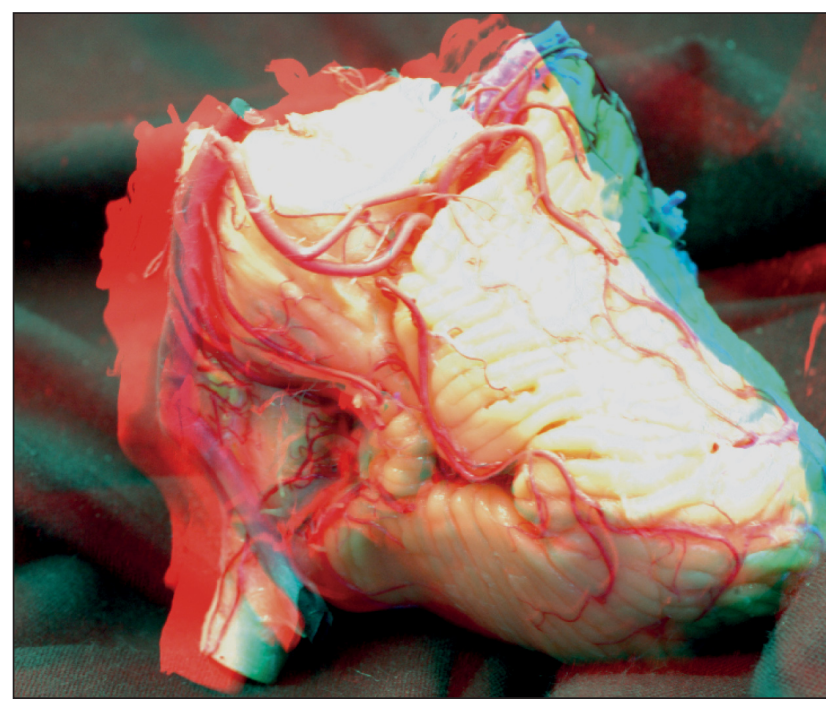

Figura 8. Visão estereoscópica da peça anatômica mostrando os ramos da artéria basilar.

trigêmeo na ponte. Esse segmento emerge da margem anterolateral do tronco até a margem anterior da fissura cerebelo-mesencefálica. Junto a esse segmento, encontram-se a veia basal e a artéria cerebelar inferior posterior, que correm acima deste ${ }^{18,19}$.

Em metade dos casos existe uma zona de contato da ACS com o trigêmeo, que se estende de 3 a $4 \mathrm{~mm}$ na superfície do nervo, que pode estar associado a um quadro clínico de neuralgia trigeminal. Assim como o tronco principal, o tronco rostral e o caudal também emitem ramos perfurantes, geralmente do tipo circunflexo e destinam-se a duas áreas principais: a junção dos pedúnculos superior e médio e abaixo do sulco, entre os colículos superiores e inferiores da lâmina quadrigêmea ${ }^{18,19}$.

\section{Segmento cerebelo-mesencefálico}

Trata-se de uma porção tortuosa da artéria que cursa dentro da fissura cerebelo-mesencefálica. Nessa fissura, a ACS dá origem aos "ramos hemisféricos", cujo número é variável, mas que em média costumam ter três ramos: medial, intermédio e lateral. $\mathrm{O}$ ramo medial tem sua origem normalmente do tronco rostral da ACS e o lateral, do tronco caudal. Esses ramos correm até a superfície tentorial, onde se ramificam de um a sete ramos e terminam entre as folhas do cerebelo ${ }^{20,21}$.

Dos ramos hemisféricos nascem as artérias pré-cerebelares, que formam dois grupos: o "medial" e o "lateral". O grupo medial é formado por artérias pequenas que cursam entre o véu medular superior e o lóbulo central, e o grupo

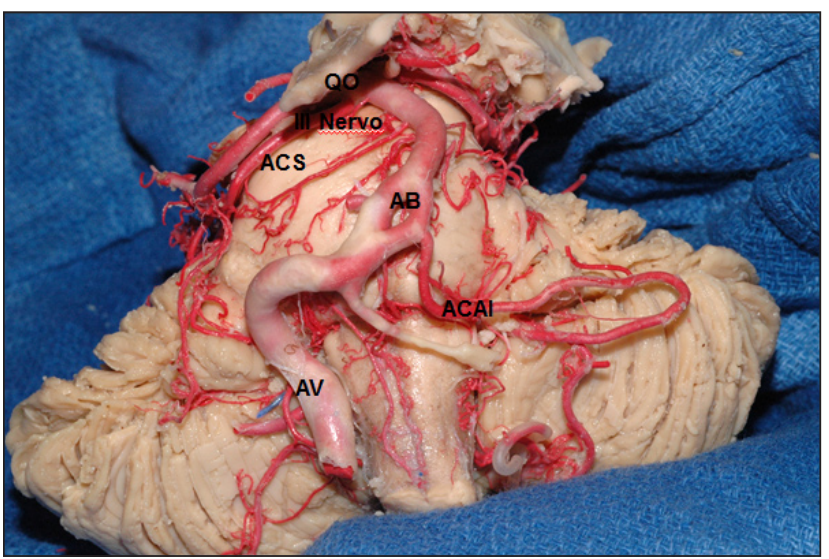

Figura 9. Visão anterior do tronco cerebral evidenciando a vascularização da fossa posterior. AV: artéria vertebral; $\mathrm{AB}$ : artéria basilar (observa-se fenestração); $\mathrm{ACIA}$ : artéria cerebelar inferior anterior; IIICN: nervo oculomotor; ACS: artéria cerebelar superior; QO: quiasma óptico.

lateral que cursa entre os pedúnculos superior e médio e as asas do lóbulo central. Essas artérias irrigam a porção do córtex lateral ao vermis, os núcleos cerebelares profundos e o colículo inferior ${ }^{20,21}$.

\section{Segmento cortical}

A porção terminal da ACS, o segmento cortical, corresponde às artérias que correm pelo córtex cerebelar junto à superfície inferior da tenda do cerebelo. Este segmento emite ramos corticais que costumam ser responsáveis pela irrigação da superfície tentorial e a porção superior da superfície petrosa adjacente ${ }^{22}$. O padrão mais frequente dessas artérias é a emissão de três ramos hemisféricos (lateral, intermediário e medial) que irrigam, na sua superfície, as porções adjacentes dos hemisférios cerebelares, voltada para a tenda do cerebelo; e, duas artérias vermianas, uma que corre pela linha média e outra que se dirige à superfície tentorial ${ }^{22}$. É comum ainda a existência de um ramo marginal, em metade dos indivíduos, que irá irrigar parte da superfície petrosa, próximo da superfície tentorial, originado do segmento ponto-mesencefálico lateral, seguindo em direção à superfície cortical ${ }^{22}$.

\section{Artéria cerebelar inferior anterior}

A ACIA é um ramo da artéria basilar que costuma ter origem na metade inferior dessa artéria, ao nível do sulco bulbo-pontino. Após sua emergência, ela permanece em íntima relação com o nervo abducente, corre em direção 
lateral junto ao sulco bulbo-pontino, passando entre o ponto de origem dos nervos glossofaríngeo, abaixo, e nervo facial e vestíbulo-coclear, acima.

Há algumas relações entre a ACIA e os dois nervos que correm pelo meato acústico. Neste ponto, costuma ocorrer a bifurcação da artéria em troncos rostral e caudal, semelhante ao fenômeno ocorrido na ACS. De lá, a artéria segue sua trajetória para a porção posterior do cerebelo, mas ela ainda enviará ramos que suprirão estes nervos e artérias responsáveis pela irrigação do tronco encefálico (as artérias perfurantes) e demais artérias que passarão pelo forame de Luschka (forame lateral do quarto ventrículo) para irrigar o plexo corioideo. A artéria dirige-se ao ângulo ponto-cerebelar. Deste local, ela seguirá para o flóculo, no pedúnculo cerebelar médio. Chegando à face lateral do cerebelo, ela corre, então, pela fissura cerebelar horizontal (Figuras 2 e 7 ).

Da mesma forma que a ACS, divide-se a ACIA em quatro segmentos:

\section{Segmento pontino anterior}

Este é o segmento que vai dar origem à ACIA até uma linha imaginária que passa no eixo maior da oliva inferior. Seus ramos direito e esquerdo nem sempre têm origem no mesmo nível e, nem sempre, ela nasce como um tronco único, podendo, em alguns casos, nascer como tronco duplo.

\section{Segmento pontino lateral}

Sua trajetória vai do final do segmento anterior até o flóculo. Deste segmento sairá a artéria labiríntica, a qual irrigará os nervos que entram no meato acústico interno e as artérias perfurantes recorrentes que irrigarão o tronco, assim como a artéria subarqueada, que se dirige para a fossa subarqueada. É neste ponto, após passar pelos nervos facial e vestibulococlear, que a artéria se duplica em divisões rostral e caudal, na maioria dos hemisférios estudados. Nos casos em que essa duplicação não acontece, a bifurcação ocorre antes destes nervos ou da artéria, que desde sua origem, é um tronco duplo. No entanto, mesmo nestes casos, a distribuição territorial dos vasos costuma ser semelhante ao das artérias de tronco único.

\section{Segmento floculonodular}

O segmento floculonodular vai do flóculo até o pedúnculo cerebelar médio. Nesta porção, a artéria já se encontra dividida em dois troncos. O tronco rostral localizado no flóculo que enviará ramos para o forame do teto do quarto ventrículo (Luschka), correrá para o lábio superior da fissura cerebelo-pontina e para a superfície petrosa do cerebelo. Os troncos caudais entram na fissura cerebelo-bulbar e se dirigem ao córtex cerebelar inferiormente ao flóculo.

\section{Segmento cortical}

O último dos segmentos da ACIA, o cortical, vai do pedúnculo cerebelar até a superfície petrosa do cerebelo, onde esta artéria é a principal responsável pela sua irrigação. Seu território é bastante variável e pode abranger apenas o flóculo e porções adjacentes, como pode se espalhar pela maioria da superfície petrosa, ocorrendo sobreposição do seu território com a ACS (na porção inferior da superfície petrosa) e com a ACIP, a qual irriga a parte lateral da superfície suboccipital. Devido a essa sobreposição de áreas, com frequência, os troncos rostral e caudal se anastomosam com a ACS e com a ACIP.

Há várias relações da ACIA com os nervos que correm pelo meato acústico interno. Nas proximidades do nervo facial, logo abaixo deste, surge a artéria cerebelo subarqueada, a qual corre para a fossa subarqueada e se dirige ao cerebelo, sendo a principal responsável pela irrigação do flóculo. Outro aspecto importante é o fato de que as principais artérias que irrigam os órgãos do ouvido interno são ramos da ACIA, como a artéria labiríntica, que se ramifica e termina por originar as artérias vestibular, coclear e vestibulococlear, à medida que estas chegam a seus correspondentes destinos.

Da artéria labiríntica costuma ter origem, a artéria subarqueada, ou seja, uma artéria que se origina medialmente ao meato acústico interno, penetra a dura-máter que reveste a fossa subarqueada e entra no canal subarqueado. Sua importância reside no fato de que ela irriga o osso petroso na região dos canais semicirculares e, ainda, porque ela pode servir de trajeto para disseminação, via canal subarqueado, infecções para as meninges e para o seio petroso, provenientes da região mastóidea.

\section{Artéria cerebelar inferior posterior}

A ACIP é a mais inconstante das artérias cerebelares, no que diz respeito ao percurso e ao seu território de distribuição. Ela se origina normalmente na margem posterior ou lateral da artéria vertebral, próximo à oliva, sendo o maior dos ramos da artéria vertebral (em poucos casos pode ter sua origem na artéria basilar). Sua origem pode ser abaixo do forame magno ou acima deste, mas na maioria das vezes ( $83 \%$ dos casos), essa origem se dá acima. 
Passa junto às raízes dos nervos cranianos baixos, primeiro pelo hipoglosso, podendo passar acima, abaixo ou pelo meio das suas raízes e depois pelo glossofaríngeo, vago e acessório, os quais normalmente situam-se abaixo ou no mesmo nível desta artéria. Seu trajeto é bem mais tortuoso do que o das demais artérias cerebelares: ela corre para as tonsilas cerebelares, dirige-se para o sulco cerebelobulbar e de lá, distribui-se para o vermis e para a superfície suboccipital do cerebelo (Figura 3).

Durante seu trajeto, a ACIP se divide em cinco segmentos, descritos a seguir e que nem sempre podem estar presentes.

\section{Segmento bulbar anterior}

O segmento bulbar anterior é aquele cujo início é marcado pela sua emergência, quase sempre na artéria vertebral. Diz-se que este segmento está presente apenas no caso da artéria emergir anterior ao bulbo, mas não se sua origem for lateral a esse. Sua existência costuma ocorrer na ACIP, cuja origem se dá na porção superior da artéria vertebral. Ao começar sua trajetória a partir da origem desse vaso, essa porção da artéria entra em contato com raízes do nervo hipoglosso. A partir daí, esse segmento corre no sentido posterior e o seu limite anatômico é uma linha imaginária que passa na porção mais proeminente da oliva.

\section{Segmento bulbar lateral}

O segmento bulbar lateral está presente na maioria das artérias e é aquele que vai do ponto em que a artéria passou pela porção mais proeminente da oliva indo até o ponto de emergência das raízes do glossofaríngeo, vago e acessório.

\section{Segmento tonsilobulbar}

Esse segmento se estende do ponto de emergência dos nervos cranianos IX, X e XI e segue por um trajeto posterior, até a artéria atingir a metade inferior da tonsila. No pólo inferior da tonsila, usualmente, há a bifurcação da PICA em tronco medial (menor) e tronco lateral (maior), sendo o local mais comum a fissura telovelotonsilar.

O segmento tonsilobulbar, juntamente com o telovelotonsilar (descrito a seguir), costuma enviar ramos pelo forame do teto do quarto ventrículo (Luschka), os quais desempenharão importante papel na irrigação do plexo coróide, nas porções deste que não forem irrigadas pelos ramos da ACIA. Dentro do quarto ventrículo, a ACIP irriga ainda a porção média do seu teto e a porção medial do recesso lateral.
Destes três segmentos bulbares, costuma surgir uma série de pequenas artérias chamadas artérias perfurantes, do tipo direto ou circunflexo. Elas estabelecem várias anastomoses entre si, formando um plexo, e terminam por penetrar o tronco, desempenhando, pois, um papel fundamental na irrigação do bulbo.

\section{Segmento telovelotonsilar}

O segmento telovelotonsilar é caracterizado por ser o mais complexo da PICA. Origina-se na porção ascendente junto à tonsila. No seu percurso, pode formar uma alça com curvatura convexa rostral (a alça cranial), de localização variável, indo da margem anterior à superior e da extremidade lateral até o aspecto medial do véu medular inferior. Ele penetra as fissuras existentes entre o verme, a tonsila e o cerebelo e origina ramos que irrigam a tela corioidea e o plexo corioideo do quarto ventrículo.

No caso da artéria já se encontrar bifurcada nesta topografia, o segmento medial irá alcançar o vermis, via fissura vermo-hemisférica, e o segmento lateral, correndo pela fissura telovelotonsilar para alcançar o hemisfério. Os ramos que passam pela fissura telovelotonsilar mandam ramos penetrantes, os quais irão ser os responsáveis pela irrigação do núcleo denteado.

\section{Segmento cortical}

O segmento cortical é aquele que começa após a saída da artéria do sulco delimitado pelo vermis, a tonsila e o hemisfério. Na sua distribuição pela superfície do cerebelo, há muitas áreas de sobreposição de território de irrigação entre esta artéria e a ACS.

O tronco medial, o menor dos troncos da ACIP, costuma originar um grupo de ramos corticais denominados vermianos, onde acabará essa porção da artéria. Eles se originam na fissura vermo-hemisférica. Sua principal zona de irrigação constitui o verme e uma porção de hemisfério adjacente.

O tronco lateral, o maior dos troncos da ACIP, terá dois grupos de ramos que sairão dele: os ramos hemisféricos e os tonsilares. Os ramos hemisféricos, na maioria das vezes, são em número de três. Surgem da fissura vermo-hemisférica e irrigam os segmentos medial, intermédio e lateral da superfície suboccipital. Esse território muitas vezes se confunde com o da ACS, mas é caracterizado por ser o território mais constante de irrigação da ACIP.

Finalmente, os ramos tonsilares, rotineiramente um ou dois, costumam irrigar a tonsila. Nem sempre se encontram presentes e, nesta situação, a tonsila é irrigada pelos ramos hemisféricos adjacentes. 


\section{Discussão}

Em relação aos aspectos clínico-cirúrgicos, a oclusão da ACS é incomum, mas quando ocorre gera quadros clínicos muito ricos. A sintomatologia compreende vômitos, vertigem súbita, incapacidade para ficar de pé e deambular (quadro este que é compartilhado com as outras oclusões de artérias cerebelares), síndrome de Horner contralateral, nistagmo, perda de termoanalgesia contralateral, transtorno da audição contralateral ${ }^{23-25}$.

Observa-se que na oclusão dessa artéria, a sobrevivência e manutenção da viabilidade cerebelar dependerão da circulação colateral, mas infelizmente, sua área é particularmente susceptível à diminuição da irrigação por ser ponto limítrofe entre a irrigação das artérias vertebrais e a da artéria basilar, sendo que uma limitação de fluxo da artéria vertebral ipsilateral, mesmo na ausência de oclusão da ACS, pode levar ao infarto deste território ${ }^{26,27}$.

São muitas as vias cirúrgicas para se abordar a ACS. A primeira é a craniotomia temporal, com levantamento do lobo occipital e temporal e incisão na tenda do cerebelo, que possibilita uma abordagem supratentorial e subtemporal. Esta técnica cirúrgica pode ser usada quando se quer obter exposição da ACS na sua origem e nos três primeiros segmentos (ponto-mesencefálico anterior e lateral e cerebelo-mesencefálico $)^{24,25}$.

Outra forma de se ter acesso cirúrgico à ACS é através de craniotomia pterional, que é usada para acessá-la em casos nos quais se encontra localizada acima do dorso da sela. Contudo, no caso de sua origem ser baixa, podese utilizar abordagem petrosa. A craniotomia suboccipital lateral é utilizada para se obter acesso à artéria quando esta se encontra na fissura cerebelo-mesencefálica ou próxima ao nervo trigêmeo ${ }^{28,29}$.

Em relação à ACIA, dentre as afecções que acometem este território, a mais comum é o desenvolvimento de tumores do ângulo ponto-cerebelar, principalmente schwannomas do acústico, sendo raros os aneurismas e malformações nesta $\operatorname{artéria}^{30}$.

Devido à sua intrínseca relação com os nervos cranianos VII e VIII, uma síndrome compressiva causada pela oclusão da ACIA se apresenta com quadro de paralisia facial, vertigem, surdez, náuseas e vômitos, associados à ataxia e discinesia. Destes sintomas, a vertigem costuma ser o sintoma mais importante. Em geral, são sintomas de origem súbita, sem perda de consciência. Outras conexões entre núcleos de nervos originam também quadros de déficits termoalgésicos faciais homolaterais (nervo trigêmeo). Déficits termoalgésicos também são vistos na metade contralateral, pelo acometimento de parte das fibras do trato espinotalâmico lateral ${ }^{30}$.

Cabe ressalvar que devido à intensa variabilidade da anatomia da ACIA, associada à existência ou não de circulação colateral, nem sempre os mesmos sintomas acometem os pacientes de uma maneira uniforme. $\mathrm{O}$ acesso cirúrgico da ACIA ocorre de acordo com a localização da lesão que se almeja tratar, seguindo o seguinte esquema:

- acesso suboccipital lateral que é usado para lesões nos segmentos meatal e pós-meatal da ACIA e regiões abaixo do trigêmeo e adjacente ao meato acústico interno.

- acesso subtemporal que se usa nos casos de uma ACIA com origem alta ou medial ao nervo trigêmeo.

- acesso pré-sigmóideo que é indicado para ressecções profundas, localizadas à frente do tronco encefálico.

Uma síndrome oclusiva que acometa a ACIP quase sempre cursa com isquemia do bulbo e do cerebelo, sendo mais comum na porção dorsal ao núcleo olivar inferior. O quadro clínico costuma ser rico, em função do acometimento de raízes de vários nervos cranianos (parestesia facial homolateral, queda do palato homolateral, disartria e rouquidão, ataxia, vertigem, nistagmo, síndrome de Horner e vômitos) e das vias nervosas ascendentes do tronco (insensibilidade termoalgésica contralateral), caracterizando a "síndrome bulbar lateral".

A causa mais comum é a oclusão por ruptura de placa aterosclerótica e trombose subsequente. Oclusões arteriais que ocorrem distais aos ramos bulbares produzem uma síndrome semelhante à labirintite, devido ao acometimento do complexo floculonodular, mas nestes casos, os achados resultantes de lesões do tronco cerebral estão ausentes ${ }^{27}$.

\section{Conclusão}

O estudo anatômico das artérias infratentoriais, sob a perspectiva estereoscópica, fornece um rico detalhamento da anatomia microcirúrgica para o cirurgião, pelo fato de que, uma região anatômica pode ser estudada sobre diferentes ângulos e de acordo com a posição cirúrgica dos pacientes. A documentação estereoscópica é útil ao fornecer uma noção de profundidade da anatomia documentada. Estudos deste tipo são fundamentais para colocar o cirurgião em um nível mais avançado da curva de aprendizado, previamente à realização de procedimentos complexos nos pacientes.

\section{Referências}

1. Isolan GR, Rowe R, Al-Mefty O. Microanatomy and surgical approaches to the infratemporal fossa: an anaglyphic three-dimensional stereoscopic printing study. Skull Base. 2007;17(5):285-302. 
2. Isolan GR, Chem CC, Webster R, et al. Reconstrução da base do crânio - enxertos e retalhos regionais - duas séries diferentes provenientes de um Departamento de Neurocirurgia e de um Departamento de Cirurgia Plástica. J Bras Neurocir. 2007;18(1):5-13.

3. Reisch R, Vutskits L, Patonay L, et al. The meningohypophiseal trunk and its blood supply to different intracranial structures: an anatomical study minim invasive. Minim Invasive Neurosurg. 1996;39(3):78-81.

4. Testut L, Latarjet A. Tratado de anatomia humana. Madrid: Salvat Editores S.A; 1988. p.206-40.

5. Rhoton Jr AL. The cavernous sinus, the cavernous venous plexus, and the carotid collar. Neurosurgery. 2002;51(4 Suppl):S375-410.

6. Rhoton Jr AL. Supratentorial arteries. Neurosurgery. 2002;51 (4 Suppl 1):S53-120.

7. Yasargil MG. From the microsurgical laboratory to the operating theatre. Acta Neurochir (Wien). 2005;147(5):465-8.

8. Ribas GC, Bento RF, Rodrigues Jr AJ. Anaglyphic three-dimensional stereoscopic printing: revival of an old method for anatomic and surgical teaching and reporting. I Neurosurg. 2001;95(6):1057-66.

9. Isolan GR, Ribas Filho JM, Malafaia O, et al. Técnicas de anastomoses microvasculares em ratos no laboratório de microcirurgia do IMPEM. Rev Med Paraná. 2005;62(2):19-23.

10. Isolan GR, Oliveira E, Recalde R. Microanatomical study of the choroidal fissure in ventricular and cisternal approaches. Arq Neuropsiquiatr. 2005;63(3B):801-6.

11. Yasargil MG. A legacy of microneurosurgery: memoirs, lessons, and axioms. Neurosurgery. 1999;45(5):1025-92.

12. Isolan GR. A construção do conhecimento pelo jovem neurocirurgião: ética, ciência, filosofia e a importância do treinamento em laboratório de microcirurgia. J Bras Neurocir. 2009;20(3):314-34.

13. Siang CC. Microanatomia do seio cavernoso. [tese]. São Paulo: Universidade Federal de São Paulo/Escola Paulista de Medicina, 1992.

14. Parkinson D. A surgical approach to the cavernous portion of the carotid artery. Anatomical studies and case report. J Neurosurg. 1965;23(5):474-83.

15. Inoue $\mathrm{T}$, Rhoton Jr AL, Theele $\mathrm{D}$, et al. Surgical approaches to the cavernous sinus: A microsurgical study. Neurosurgery. 1990; 26:903-32.

16. Yasargil MG, Cravens GF, Roth P. Surgical approaches to "inaccessible" brain tumors. Clin Neurosurg. 1988;34:42-110.

17. Isolan GR, Krayenbuhl N, Oliveira E, et al. Microsurgical anatomy of the cavernous sinus: measurements of the triangles in and round it. Skull base. 2007;17(6):357-67.

18. Aguiar PHP, Veiga JCE, Tahara A, et al. Posterior inferior cerebellar artery aneurysm: skull base approach and technical challenges. Asian J Surg. 2008;2:35-44.
19. Harris FS, Rhoton Jr AL. Anatomy of the cavernous sinus: a microsurgical study. J Neurosurg. 1976;45(2):169-80.

20. Isolan GR, Oliveira E, Mattos JP. Microsurgical anatomy of the arterial compartment of the cavernous sinus: analysis of the 24 cavernous sinus. Arq Neuropsiquiatr. 2005;63(2A): 259-64.

21. Rhoton Jr AL. Anatomy of saccular aneurysms. Surg Neurol. 1980;14:(1)59-66.

22. Tran-Dinh H. Cavernous branches of the internal carotid artery: anatomy and nomenclature. Neurosurgery. 1987;20(2): 205-10.

23. Rhoton Jr AL. Anatomic foundations of aneurysm surgery (honored guest lecture). Clin Neurosurg. 1994;41:289-324.

24. Rhoton Jr AL. Aneurysms. Neurosurgery. 2002;51(4 Suppl 1):S121-58.

25. Yasargil MG. Microneurosurgery. Stuttgart: Georg Thieme Verlag; 1996.

26. Yasargil MG. Reflections of a neurosurgeon. Clin Neurosurg. 1988;34:16-21

27. Yasargil MG, Chandler WF, Jabre AF, et al. Neurosurgical horizons. Clin Neurosurg. 1988;34:22-41.

28. Mussi A, Rhoton Jr AL. Telovelar approach to the fourth ventricle: microsurgical anatomy. J Neurosurg. 2000;92(5):812-23.

29. Pollack IF, Polinko P, Albright L, et al. Mutism and pseudobulbar symptoms after resection of posterior fossa tumors in children: incidence and pathophysiology. Neurosurgery. 1995;37(5): 885-93.

30. Rhoton Jr AL. Microsurgical anatomy of posterior fossa cranial nerves. In: Barrow DL (editor). Surgery of the cranial nerves of the posterior fossa: neurosurgical topics. Chicago, AANS; 1993. p.1-103.

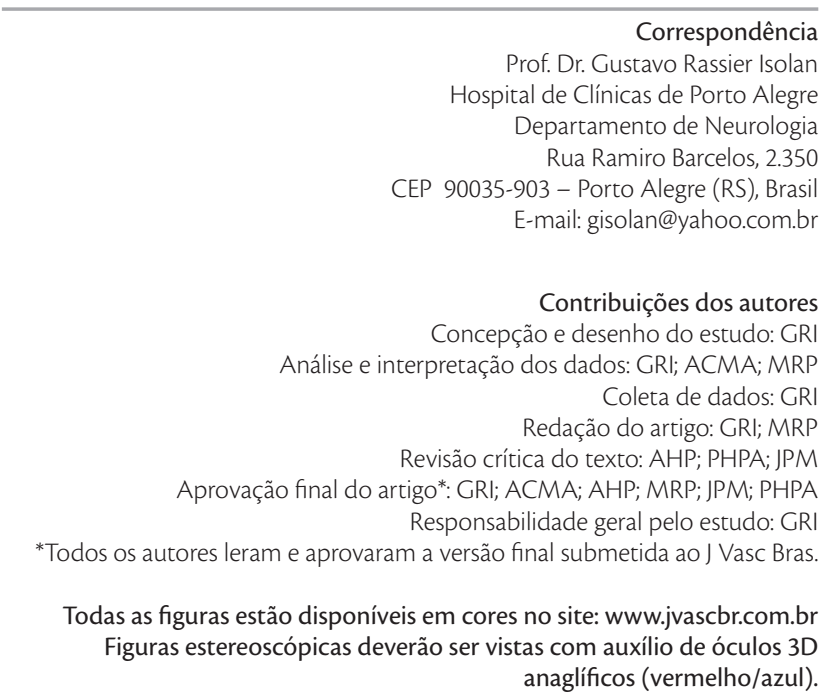

